Musées, Patrimoine et Culture scientifiques et techniques

$171 \mid 2017$

mai-juin 2017

\title{
Le Quai des Savoirs, un nouveau lieu pour la Culture scientifique, technique et industrielle
}

\section{François Lajuzan}

\section{OpenEdition \\ Journals}

Édition électronique

URL : http://journals.openedition.org/ocim/1783

DOI : $10.4000 /$ ocim. 1783

ISSN : 2108-646X

\section{Éditeur}

OCIM

\section{Édition imprimée}

Date de publication : 1 mai 2017

Pagination : 29-33

ISSN : 0994-1908

\section{Référence électronique}

François Lajuzan, « Le Quai des Savoirs, un nouveau lieu pour la Culture scientifique, technique et industrielle », La Lettre de l'OCIM [En ligne], 171 | 2017, mis en ligne le 01 mai 2018, consulté le 03 mai 2019. URL : http://journals.openedition.org/ocim/1783 ; DOI : 10.4000/ocim.1783

Ce document a été généré automatiquement le 3 mai 2019.

Tous droits réservés 


\section{Le Quai des Savoirs, un nouveau lieu pour la Culture scientifique, technique et industrielle}

\section{François Lajuzan}

L'entrée du Quai des Savoirs.

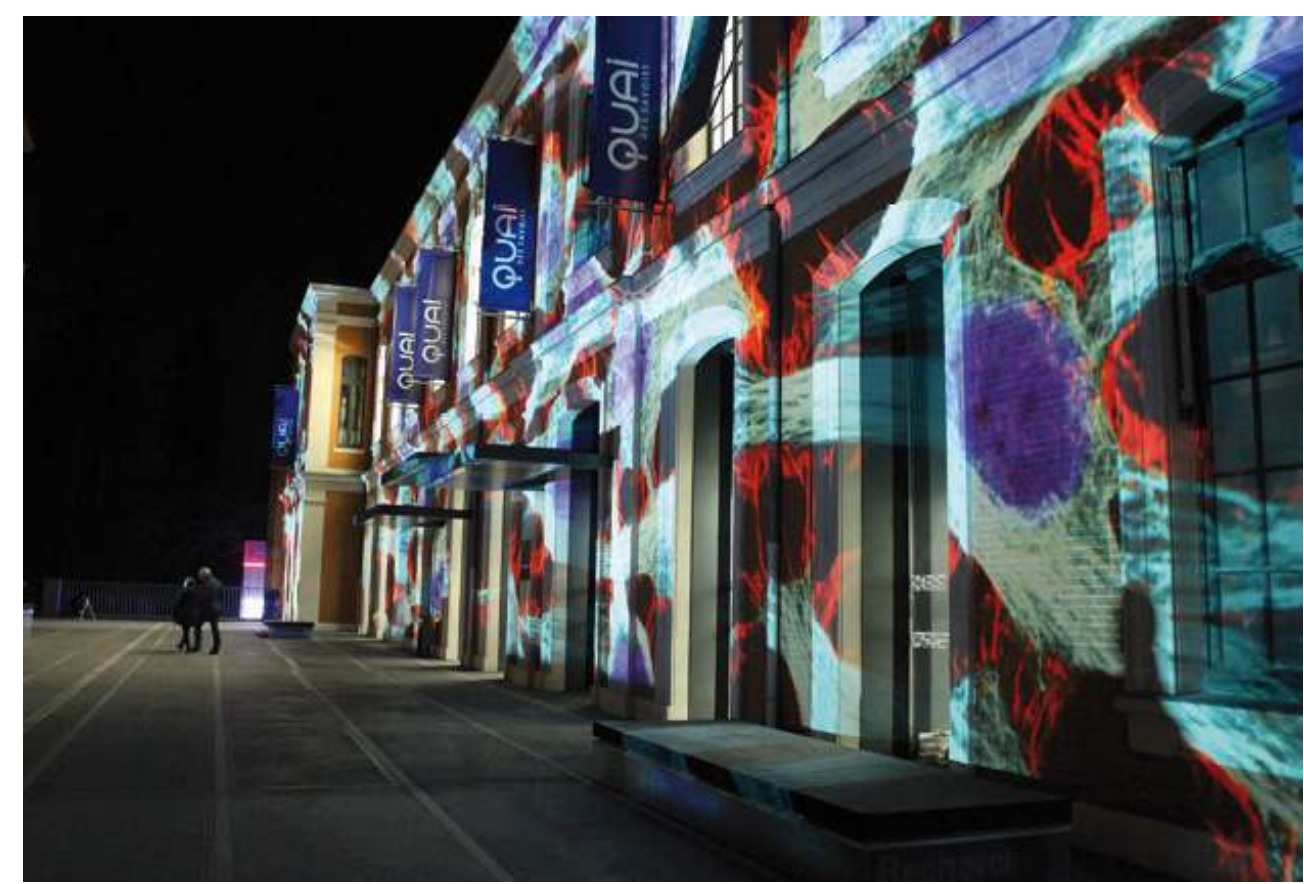

(c) DCSTI Toulouse Métropole

Le 16 février 2016 à Toulouse, le Quai des Savoirs ouvrait ses portes au public. Expositions interactives dans la grande salle de $760 \mathrm{~m}^{2}$, espace pour les moins de sept ans (le Quai des Petits), Hall des Manips, réalité augmentée à l'entrée, cafés débats au bistrot, centre de loisirs scientifique pour les 8-12 ans, Petites Manips du week-end à expérimenter en 
famille, "Nocturnes" avec des artistes... Tous les ingrédients d'un centre de culture scientifique, technique et industrielle sont réunis pour faire de ce lieu le rendez-vous incontournable des amateurs de recherche, d'innovation et de technologie, ouvert également aux sciences humaines et sociales.

2 Sur $6000 \mathrm{~m}^{2}$ dont la moitié ouverts au public, le Quai des Savoirs vient combler un manque dans une métropole marquée depuis des siècles, par la volonté de connaissance et le dynamisme industriel. Le muséum d'Histoire naturelle avait ouvert la voie, il y a 150 ans, puis la Cité de l'Espace et plus récemment Aeroscopia (musée aéronautique de Toulouse Blagnac) ont étoffé l'offre, avec un succès considérable. Le Quai des Savoirs vient compléter cette offre avec une approche pluridisciplinaire.

\section{Un lieu atypique et fédérateur}

3 Le Quai des Savoirs n'est pas un CCSTI tout à fait comme les autres. Sa genèse autant que sa réalisation ont procédé d'une démarche partenariale forte, associant les équipements existants, les associations de CSTI, les services publics, les universités rassemblées au sein de l'université Fédérale (COMUE), le CNRS, l'INRA, les laboratoires, écoles d'ingénieurs et clubs d'entreprises. De cet ensemble a émergé peu à peu, au fil des municipalités, un projet cohérent dans lequel chacun trouve sa place tout en continuant à mener son action spécifique. C'est ainsi que tous se retrouvent, avec le Rectorat, la DRRT (Délégation Régionale à la Recherche et à la Technologie) et la Région, au sein d'un Comité d'Orientation, chargé de tracer les lignes de force du Quai autour du leitmotiv "Construire l'avenir".

$480 \%$ des actions et activités sont apportées et gérées par les différents partenaires, à charge pour le pilote de chacune d'y associer les autres acteurs CSTI. C'est ainsi que l'université Fédérale programme et organise une soixantaine de cafés débats par an avec des chercheurs et doctorants, Science Animation pilote les dispositifs du hall d'entrée et du Hall des Manips avec des laboratoires de recherche, Planète Sciences gère le centre de loisirs, le Muséum a réalisé la première grande exposition...

Ce système est dirigé et coordonné par la Direction de la Culture scientifique, technique et industrielle de Toulouse Métropole, une équipe réduite qui crée le lien et la cohérence entre les diverses propositions, gère en direct certaines activités et coordonne la programmation. Quant à l'exploitation (technique, logistique, sécurité, informatique, administration et finances, communication, juridique...) elle est confiée à une équipe mutualisée avec le Muséum, qui assure ainsi ces fonctions pour les deux équipements.

6 Au total, près de deux cents actions différentes sont proposées sur l'année, allant de la simple rencontre avec un chercheur jusqu'aux grands événements de l'automne : Nuit Européenne des Chercheurs, Fête de la Science, Conférence technologique internationale Emtech organisée par le MIT Technology, Futurapolis, le festival "Lumière sur le Quai"... Et déjà sont programmées deux grandes expositions, produites par la Quai mais réalisées par les partenaires : Inventer la ville... dont vous êtes les héros de février à août 2017 (réalisation Science Animation) et une exposition inédite sur l'humanité de demain en 2018 (réalisation muséum). 


\section{Un projet territorial}

7 Le Quai des Savoirs ne limite pas son action au 39 allées Jules Guesde, bâtiment du XIX siècle qui abritait la faculté des sciences de Toulouse. Il s'agit avant tout d'un projet métropolitain, dont le but est, au-delà des actions présentées dans le bâtiment, de porter la CSTI sur les trente sept communes de Toulouse Métropole, de la plus grande (Toulouse) à la plus petite (Mongouzil, 300 et quelques habitants).

8 Pour cela, le secteur "Réseau" travaille avec différents "relais savoirs". Ces relais peuvent être des professionnels de la programmation et de l'action culturelle, de l'éducation, de l'animation socio-culturelle, ou de la politique de la ville au sein de chaque ville ou quartier de la métropole. Dans une démarche de transversalité, ils favorisent le développement des actions de CSTI sur leur territoire, en mettant en synergie les différents équipements, publics, associations, structures concernées de leur ville. Ils ont en charge la mobilisation des habitants et des publics spécifiques. Ils sont garants de la mise en cohérence de la programmation du Quai des Savoirs avec les autres événements et programmations culturelles sur leur ville. La réalisation concrète de ces actions est confiée aux associations partenaires Planète Science et Les Petits Débrouillards. Quant à l'association Science Animation, elle fait tourner sur différents territoires, Le Propulseur, un Fablab itinérant réalisé dans le cadre du programme Inmediats. L'objectif étant, à plus long terme, d'accompagner les communes dans la définition et la mise en œuvre de leur propre politique de CSTI au plus près des habitants.

9 Deux axes de travail prioritaire ont été définis :

10 - Une action volontariste en faveur de la médiation CSTI dans les quartiers prioritaires de Toulouse et plusieurs communes de banlieue. Il s'agit là de co-construire avec les habitants et acteurs locaux des actions de médiation participatives.

11 - Faire émerger dans cette zone un quartier créatif autour de la science et de l'innovation dans ce véritable "Quartier des sciences" que dessinent les allées Jules Guesde. Sur quelques centaines de mètres se succèdent université Fédérale, Quai des Savoirs, Faculté de Médecine, Muséum, Théâtre Sorano, et le Village de l'Innovation du Crédit Agricole qui a ouvert ses portes en janvier 2017. Sans oublier le Jardin des Plantes et le jardin botanique. Sous l'égide du Quai des Savoirs, une première réunion a posé les bases d'un travail commun.

\section{Un carrefour des médiations}

12 L'autre ambition du Quai des Savoirs est d'être le point de rencontre entre un maximum d'initiatives visant à créer le lien entre la CSTI et la population. Pour cela point d'exclusivité : une entreprise peut apporter sa pierre à l'édifice aux côtés d'un laboratoire, d'un enseignant ou d'une association, en lien avec des artistes en résidence de création ou avec des citoyens participant à une expérience de science participative.

Le secteur "Carrefour" gère ainsi le Quai des Petits, considéré non comme un simple lieu d'activité mais comme un terrain d'expérimentation pour une médiation CSTI dès le plus jeune âge. Les formations pour les enseignants et médiateurs trouvent également leur place au Quai des Savoirs, en associant par exemple aussi bien l'École des Docteurs que la Maison pour la Science ou les fédérations d'éducation populaire. 
Le Quai des Petits

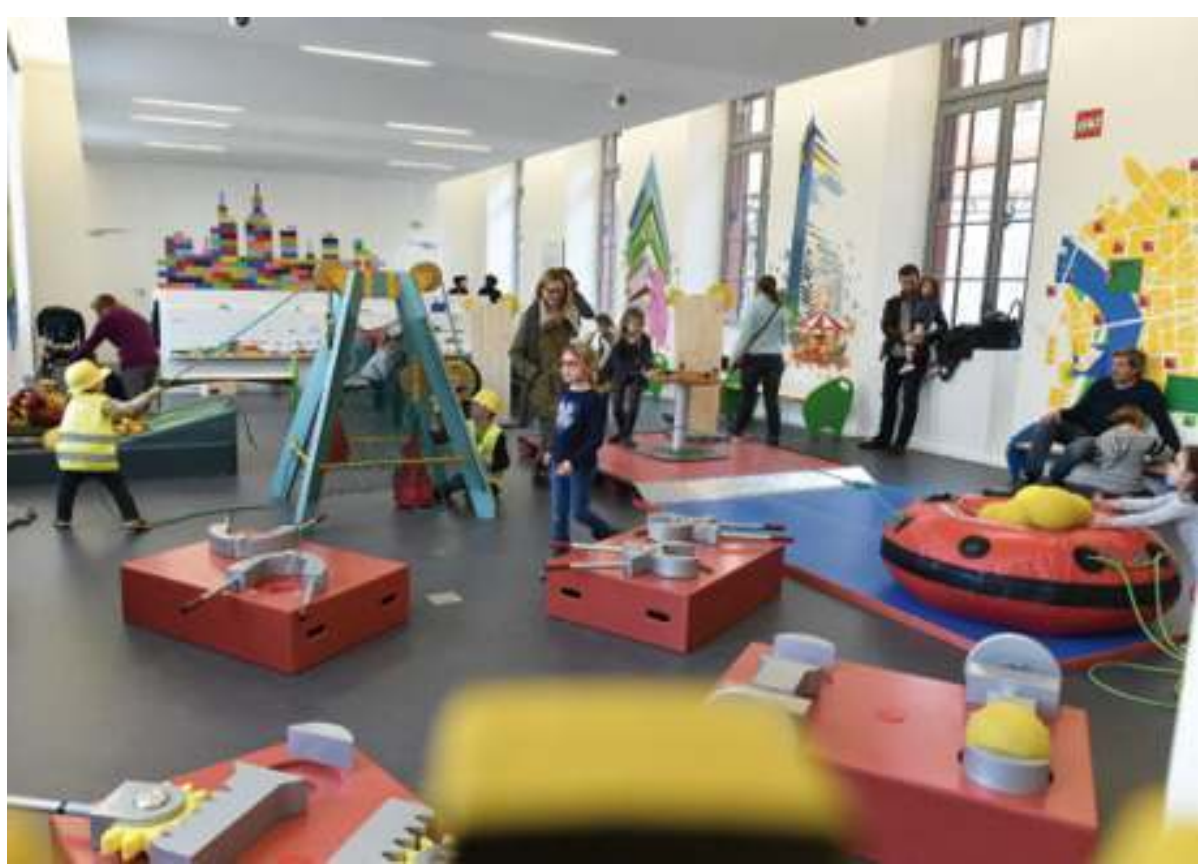

(c) Joachim Hocine plateau d'expérimentation, dans lequel Oculus Rift, Fablab, Living Lab et autres Laboratoires des Usages pourront inventer et tester de nouveaux contenus et dispositifs avec les publics.

Enfin, le Quai des Savoirs accueille dans ses murs le délégué "French Tech Toulouse" et "La Mêlée", importantes associations dédiées au numérique et à tous ses usages, offrant espaces de co-working, "Cantine Numérique", formations, débats, ateliers... Le Quai est ainsi une ruche, un lieu de foisonnement et d'émergence d'idées et de réalisations tous azimuts.

\section{Vers une Métropole des Savoirs}

Les maîtres mots de l'action du Quai des Savoirs sont la diffusion et le partage de la CSTI. Un partage dans les deux sens, car les savoirs ne sont pas tous entre les mains des chercheurs et des ingénieurs. Nous devons diffuser les dernières avancées de la recherche et de la technique, mais également être attentifs aux savoirs et aux cultures des citoyens, répondre à leurs interrogations, mettre à jour les enjeux de notre société.

Pour cela, Toulouse Métropole se dote d'une politique de CSTI dont la finalité est clairement exprimée : il s'agit de développer l'esprit critique, de donner à la personne les outils de construction de sa propre opinion instruite, sur le monde et les idées, face aux dispositifs de manipulations et de croyances largement diffusés dans nos sociétés. La finalité énoncée par la politique CSTI de Toulouse Métropole est de poser les bases et de donner les outils pour une citoyenneté active et éclairée.

Cet objectif ambitieux passe par un travail constant à l'interne comme à l'externe : 
19 - au niveau local, il s'agit d'assurer un maillage du territoire pour que la CSTI soit présente partout et au plus près des citoyens, dans une démarche de partenariat avec les forces vives et les acteurs des territoires ;

20 - au niveau national et international, il s'agit de porter l'identité "Métropole des Savoirs" comme un marqueur identitaire de la métropole, et ce jusqu'au niveau mondial.

21 Dans les deux cas, l'objectif ne sera atteint qu'en fédérant les acteurs CSTI autour de cette ambition. Concrètement, les équipements sous la responsabilité de la Métropole commencent à élaborer un programme d'actions commun. Cité de l'espace, muséum, Piste des Géants, Quai des Savoirs, peuvent être les ressorts d'un élan exceptionnel de transmission des savoirs et de la connaissance. Son impact sera d'autant plus fort si on y adjoint d'autres acteurs majeurs de la vie culturelle toulousaine, comme Aéroscopia, l'université Fédérale et toutes ses composantes, le Bazacle, les entreprises, les artistes passionnés par le sujet, et les associations de CSTI.

Plusieurs pistes de développement sont à l'étude : développement du tourisme scientifique et industriel (avec So Toulouse, marque de rayonnement touristique de Toulouse), formation de journalistes scientifiques (avec l'école de journalisme), coproductions internationales, communication commune...

23 Cette identité de Métropole des Savoirs prendra corps en 2018, grâce à l'accueil d'ESOF (Euroscience Open Forum : biennale européenne des sciences créée en 2004. Elle est le plus grand forum interdisciplinaire d'Europe). Elle se développera également dans toutes les actions mises en place au titre de la nomination de Toulouse comme Cité Européenne de la Science cette même année. La Métropole a ainsi pour ambition de développer sur l'ensemble du territoire, une programmation innovante autour de la thématique "Science dans la ville".

24 Le Quai des Savoirs prendra ainsi toute sa place au cœur du dispositif, non pas en tant que simple équipement, mais en tant qu'espace fédérateur de construction partagée pour une Métropole des Savoirs pas si utopique qu'on pourrait le croire.

\section{Rencontre avec Francis Duranthon, directeur du muséum de Toulouse}

\section{Le muséum de Toulouse : un partenaire incontournable?}

Deuxième muséum de France après celui de Paris, le muséum de Toulouse, situé tout près $\mathrm{du}$ Quai des Savoirs, se positionne depuis sa refondation en 2008, comme un acteur majeur de la Culture scientifique à Toulouse, en France et à l'international. Lieu de conservation du patrimoine (2,5 millions d'objets composent ses collections), de médiation avec les publics et plate-forme de débats sur les relations Homme - Nature Environnement, le muséum est un espace dédié à la connaissance du temps long, le temps du vivant et des grandes aires géologiques. Le Quai des Savoirs complète les offres du muséum en apportant sur le quartier une dimension technique et technologique, une vision anticipée du futur. 


\section{Quel est son périmètre d'actions au Quai des Savoirs?} Intervenant dans son comité éditorial, il participe collégialement avec les acteurs institutionnels et associatifs à la cohérence et à la variété des offres proposées ainsi qu'à l'identité des différents lieux. Il est également force de proposition et "chef de projet" sur certaines grandes expositions du Quai des Savoirs (Sacrée Science ! en 2016 et prochaine exposition de 2018) en alternance avec l'association Science Animation.

L'exposition Sacrée Science était présentée lors de l'ouverture du Quai des Savoirs.

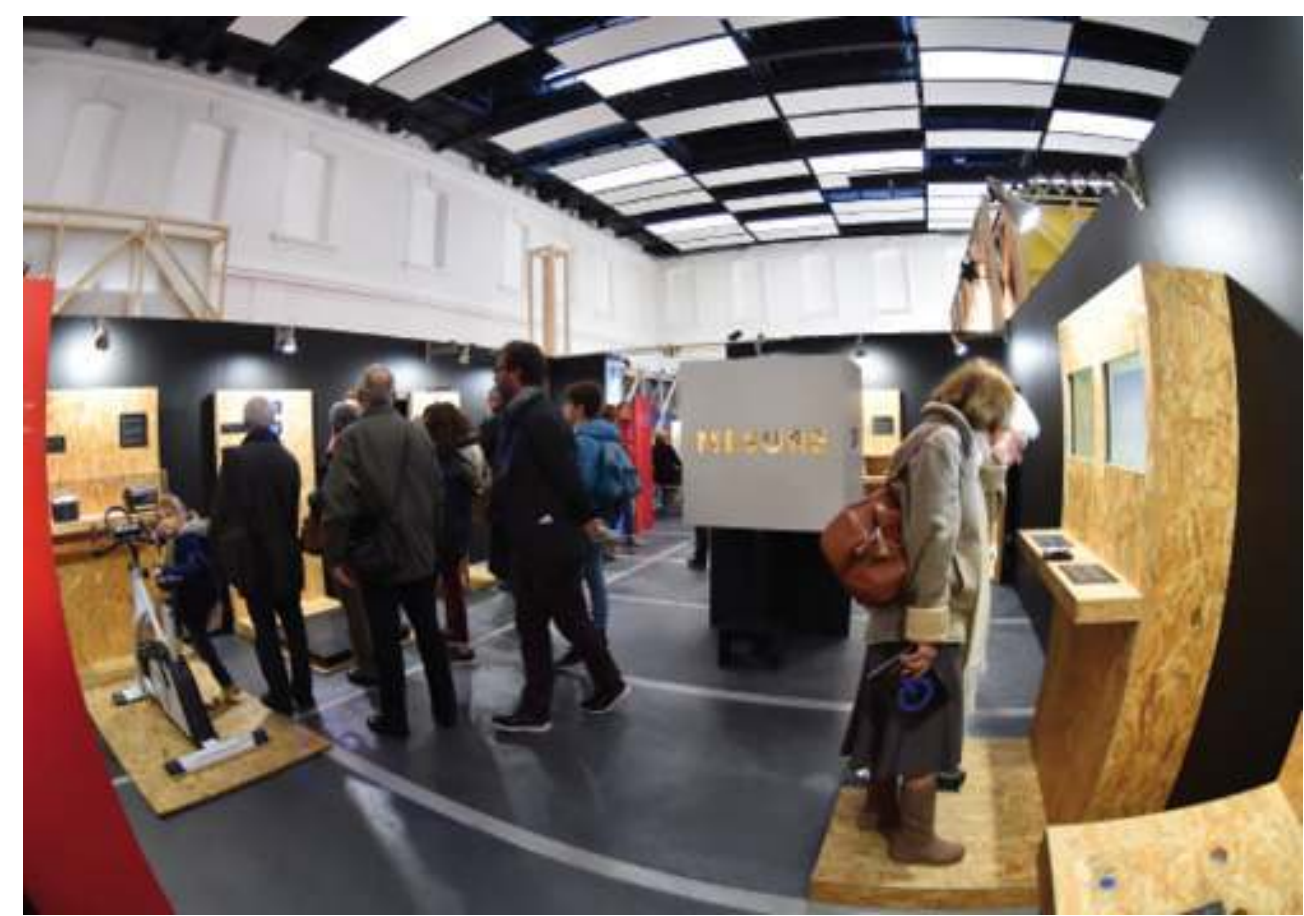

(c) Joachim Hocine

Le texte suivant figure en encadré dans l'édition papier : 


\title{
Rencontre avec Johann Langot, directeur de l'association Science Animation
}

\author{
Parlez-nous de Science Animation...
}

Depuis plus de 30 ans, l'association Science Animation œuvre pour mettre à portée de tous la science, la technique et l'innovation. Avec le concours de partenaires scientifiques, industriels et éducatifs, elle conçoit des expositions, des animations ou encore des événements. Aujourd'hui implantée au Quai des Savoirs, elle en est un des acteurs privilégiés, participant activement à ses orientations et à son animation.

\section{Quelles sont vos responsabilités et missions au Quai des Savoirs?}

Nous gérons plusieurs espaces du Quai : le Hall d'accueil où nous présentons et testons auprès du public des dispositifs interactifs de découverte des sciences et le Hall des manips, où nous proposons tous les trimestres une exposition interactive pour découvrir les coulisses des laboratoires et entreprises innovantes du territoire. Nous gérons également le "Fab Lab" et le "Living lab" où nous appréhendons et partageons avec les publics la démarche de l'innovation ouverte. Enfin, une année sur deux, nous gérons la grande salle d'exposition. Le Quai des Savoirs constitue ainsi pour Science Animation un défi de taille, à la fois passionnant et stimulant.

Le Hall des manips piloté par Science Animation.

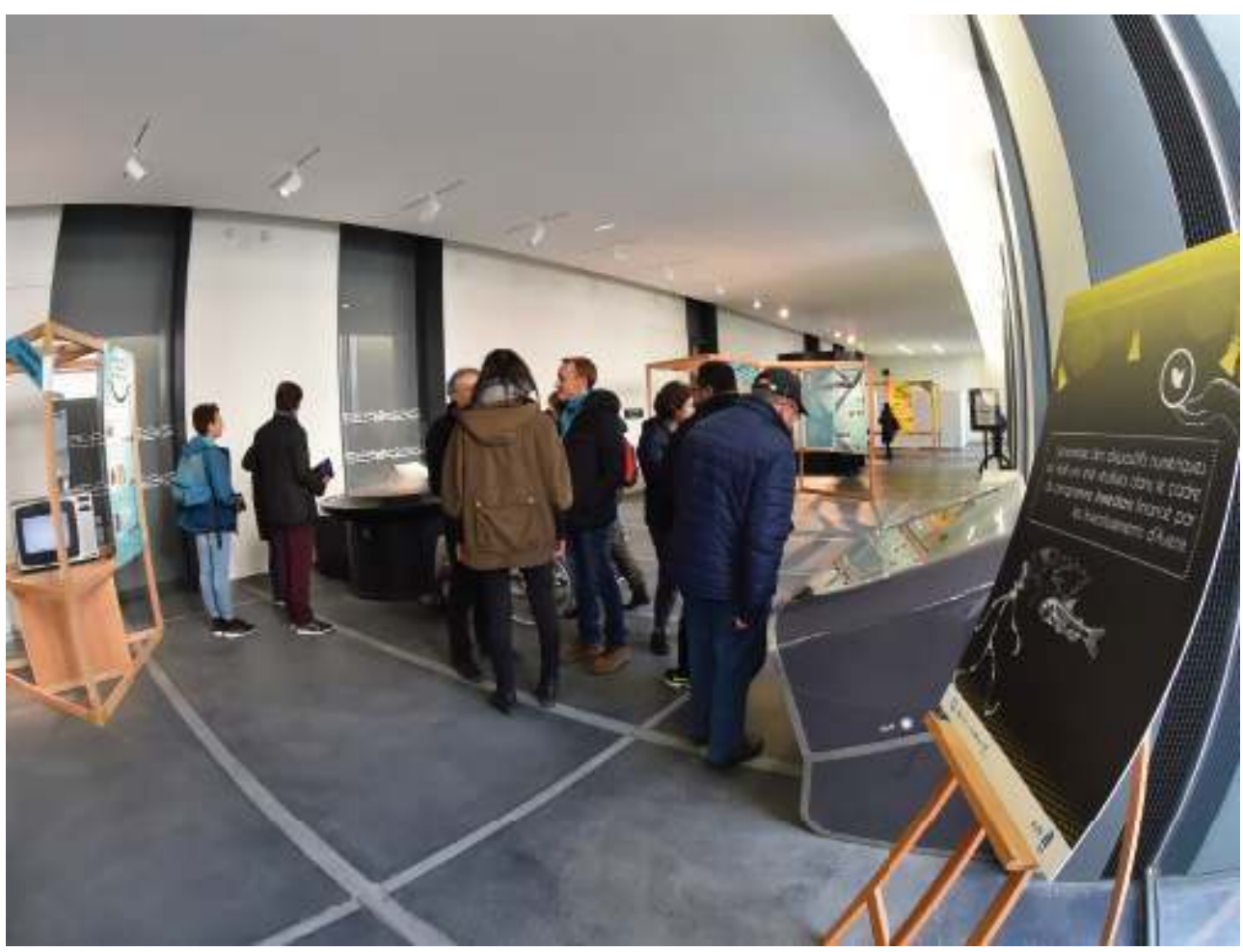

(c) Joachim Hocine 


\section{Rencontre avec Catherine Gadon, directrice du service de diffusion de la Culture des sciences et des techniques de l'université Fédérale de Toulouse}

L'université de Toulouse regroupe 29 établissements d'enseignement supérieur et de recherche. Son siège social est situé depuis 2016 dans l'un des deux anciens bâtiments des facultés de sciences situés au cœur de la Ville et entièrement réhabilités par Toulouse Métropole. L'autre bâtiment est celui du Quai des Savoirs. Cette proximité entre les deux établissements a été imaginée dès la mise en œuvre du chantier de rénovation. Sa raison essentielle tient à l'importance que jouent la science et les techniques dans l'identité même de Toulouse et plus largement de la région.

\section{Quel est le rôle de l'université au Quai des Savoirs ?}

L'université de Toulouse est naturellement présente dans la gouvernance du Quai des Savoirs avec les autres partenaires de Culture scientifique. Elle est chargée par ailleurs de coordonner des rencontres hebdomadaires au Café du Quai des Savoirs sur des questions scientifiques et pluridisciplinaires. La Mission Sauvegarde du patrimoine scientifique et technique contemporain et les différents responsables de collections universitaires sont aussi impliqués par l'apport d'éléments historiques qui aident ainsi à la vulgarisation des contenus. Enfin, elle développe en collaboration avec la direction du Quai un espace de recherche sur la CSTI qui pourra aussi être utile à l'évaluation des actions et des publics.

\section{Quels enjeux pour l'université ?}

Parce que le Quai des Savoirs est avant tout un espace de collaboration, l'université de Toulouse et ses membres sont très régulièrement sollicités pour constituer leur comité scientifique pour la réalisation d'une exposition par exemple ou pour trouver des experts d'un domaine particulier. Ce lien peut paraître banal parce qu'il s'opère déjà un peu partout sans intermédiaire, mais ici, la reconnaissance institutionnelle de cette activité permet de donner toute légitimité à l'engagement des chercheurs et offre une caution sans égal aux médiateurs scientifiques. C'est, me semble-t-il, l'enjeu important d'une telle collaboration. 
Le Café du Quai animé par des chercheurs de l'université Fédérale de Toulouse

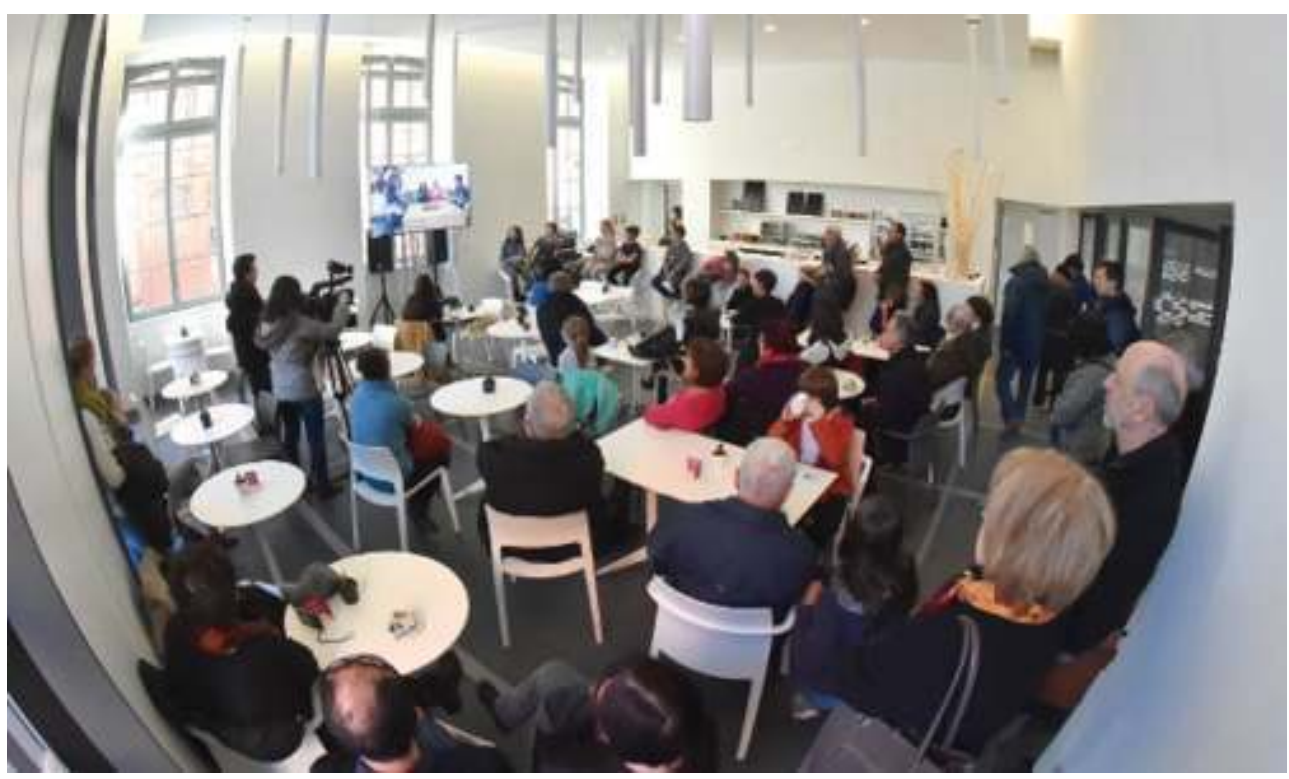

(c) Joachim Hocine

\section{Zoom sur.... l'ouverture d'un futur équipement toulousain : La Piste des Géants}

La Piste des Géants est un ensemble culturel de près de 13 hectares situé sur le site historique de Montaudran à Toulouse. Point de départ des Lignes Latécoère et de l'Aéropostale entre 1918 et 1933, les avions y étaient pilotés par des personnalités telles qu'Antoine de Saint-Exupéry ou Jean Mermoz. La Piste des Géants, qui sera entièrement ouverte au public en décembre 2018, date du centenaire du premier vol sur la Ligne, a pour ambition de faire vivre la mémoire des pionniers de l'aéronautique civile mais aussi de faire rêver à travers les créations géantes de la compagnie La Machine. Elle sera composée de quatre entités :

- "La mémoire des pionniers" : un équipement conçu autour d'un parcours scénographique avec des avions à taille réelle de près de $1000 \mathrm{~m} 2$;

- "Les jardins de la Ligne" : un parcours paysager proposant un voyage dans les ambiances végétales des pays de la Ligne de l'Aéropostale de Toulouse à Santiago du Chili ;

- "Les machines" : une grande halle animée de 150 machines conçues par François Delrozière, dont une Machine spécialement conçue pour Toulouse, le Minotaure ;

39 - "La piste" : une piste de près de $2 \mathrm{~km}$ conçue comme une grande esplanade d'événements. 
INDEX

Mots-clés : CSTI, métropole, médiation

\section{AUTEUR}

\section{FRANÇOIS LAJUZAN}

Directeur général adjoint aux Affaires culturelles de la ville de Toulouse 